\title{
Análise dos Sistemas de Controles Gerenciais e a Interface com o Ciclo de Vida de uma Indústria de Alimentos
}

\begin{abstract}
Willyam Carlos Pederssetti
Mestrado em andamento em Ciências Contábeis e Administração pela Universidade Comunitária da Região de Chapecó - Unochapecó

Administrador de Empresas

Avenida Brasil 805, Centro. São Lourenço do Oeste/SC. CEP: 89990-000

E-mail: willyam.pederssetti@gmail.com
\end{abstract}

Silvana Dalmutt Kruger

Doutorado e Mestrado em Contabilidade pela Universidade Federal de Santa Catarina -

UFSC

Professora da Universidade Comunitária da Região de Chapecó - Unochapecó

Servidão Anjo da Guarda, 295-D. Efapi. Chapecó/SC. CEP: 89809-900

E-mail:silvanak@unochapeco.edu.br

\section{RESUMO}

O tempo da transição nas empresas familiares pode motivar a utilização dos sistemas de controle gerencial (SCGs), pois um dos meios da empresa prolongar seu ciclo de vida nesses ambientes de extrema complexidade e incerteza é compreender a função desses tais sistemas. A pesquisa tem como objetivo analisar como ocorre a interface do uso dos SCGs com o ciclo de vida de uma indústria alimentícia. A pesquisa classifica-se como estudo de caso de caráter descritivo com uma abordagem qualitativa, realizado por meio de um questionário. Os resultados demonstram que a empresa está posicionada no nível de Maturidade, em seu ciclo de vida organizacional. A empresa possui uma gama de ferramentas pautadas nos SCGs, porém a maioria delas são utilizadas apenas pela alta gestão, não sendo difundidas para a utilização por outros stakeholders. Os resultados possibilitaram a construção de um diagnóstico e a sugestão de iniciativas de melhorias, sendo classificados no quadrante de Sistema de Controle Diagnóstico proposto, com oportunidades no desenvolvimento de Sistemas para Focar Busca e Atenção e estratégia de Sistemas para Formular e Implementar a Estratégia Competitiva.

Palavras-Chave: Ciclo de vida organizacional. Alavancas de controle. Empresas familiares.

\section{Analysis of Management Control Systems and the Interface With the Life Cycle of} a Food Industry

\section{ABSTRACT}

The transition time in family businesses can motivate the use of management control systems (SCGs), as one of the means for the company to have its life cycle prolonged. 
Análise dos Sistemas de Controles Gerenciais e a Interface com o Ciclo de Vida de uma Indústria

de Alimentos

Willyam Carlos Pederssetti, Silvana Dalmutt Kruger

In these environments of extreme complexity and uncertainty it seems to be vital to understand the function of such systems. The research aims to analyze how the interface of the use of SCGs occurs with the life cycle in a food industry. The research can be classified as a descriptive case study within a qualitative approach, which has made use of a questionnaire. The results demonstrate that the company is positioned at a mature level in its organizational life cycle. The company has a range of tools based on SCGs. However most of these tools seem to be used by senior management only, and are not disseminated for the use of other stakeholders. The results enabled the construction of a diagnosis and the suggestion of some improvement initiatives, being classified in the proposed Diagnostic Control System quadrant, with opportunities in the development of Systems to Focus Search and Attention and Systems strategy to Formulate and Implement the Competitive Strategy.

Keywords: Organizational life cycle. Control levers. Family businesses.

\section{Análisis de los Sistemas de Control de Gestión y la Interfaz com el Ciclo de Vida de una Industria Alimentaria}

\section{RESUMEN}

El tiempo de transición en las empresas familiares puede motivar el uso de sistemas de control de gestión (SCGs), como uno de los medios de la empresa para prolongar su ciclo de vida, en estos entornos de extrema complejidad e incertidumbre es comprender la función de estos sistemas. La investigación tiene como objetivo analizar cómo se produce la interfaz del uso de SCG con el ciclo de vida de una industria alimentaria. La investigación se clasifica como un estudio de caso descriptivo con enfoque cualitativo, realizado a través de un cuestionario. Los resultados demuestran que la empresa se posiciona en el nivel de Madurez, en su ciclo de vida organizacional. La compañía tiene una gama de herramientas basadas en SCG, sin embargo, la mayoría de ellas son utilizadas solo por la alta dirección y no se difunden para que las utilicen otras partes interesadas. Los resultados permitieron la construcción de un diagnóstico y la sugerencia de iniciativas de mejora, ubicándose en el cuadrante propuesto Sistema de Control de Diagnóstico, con oportunidades en el desarrollo de Sistemas para Enfocar Búsqueda y Atención y Estrategia de Sistemas para Formular e Implementar la Estrategia Competitiva.

Palabras-Clave: Ciclo de vida organizacional. Palancas de control. Empresas familiares. 


\section{INTRODUÇÃO}

As organizações com modelo de gestão familiar geralmente são reconhecidas como pouco propensas ao risco, elas são em sua maioria conservadoras e estagnadas, demonstrando dificuldade de ter uma visão inovadora e tecnológica (Kammerland \& Essen, 2017). Nas empresas familiares a influência da gestão familiar reflete na utilização de controles estratégicos (Oro \& Lavarda, 2019). Além disso, a utilização dos sistemas de controles gerenciais (SCG), tornam-se meios das empresas familiares prolongarem seu ciclo de vida e criar vantagens competitivas (Clinton, Mcadam, \& Gamble, 2018).

Quanto à longevidade do ciclo de vida das empresas familiares, estima-se que somente $30 \%$ dessas organizações se desenvolvem até a segunda geração, e apenas $10 \%$ sobrevivem até a terceira geração familiar, demonstrando-se uma dificuldade significativa no processo de sucessão familiar nos negócios (Hiebl, 2013; Sebrae, 2017). Entre os problemas mais comuns na gestão das empresas familiares estão a falta de estratégias definidas, os conflitos de gestão, o comportamento, a cultura organizacional, as decisões financeiras e operacionais (Bertucci, 2009), bem como a necessidade de implementação de sistemas de controles gerenciais (Carvalho, Júnior, Frezatti, \& Costa, 2010).

A cultura organizacional das empresas familiares é uma das características que distingue essas organizações das demais, primeiramente pela sobreposição dos valores familiares com os valores da empresa (Songini, Gnan, \& Malmi, 2013), posteriormente pelo processo de planejamento e gestão, especialmente quando a organização possui executivos contratados e executivos que fazem parte da família. $\mathrm{O}$ SCG pode minimizar os conflitos e as dificuldades da gestão das empresas familiares, auxiliando também no processo sucessório (Moreira \& Frezatti, 2019).

Carvalho et al. (2010), indica que o SCG sofre variações à medida que fatores internos e externos da empresa se modificam ao longo das diferentes fases do ciclo de vida organizacional. Diversos modelos definem o ciclo de vida das organizações, 
Análise dos Sistemas de Controles Gerenciais e a Interface com o Ciclo de Vida de uma Indústria

de Alimentos

Willyam Carlos Pederssetti, Silvana Dalmutt Kruger

caracterizando esse fenômeno em estágios, conforme Miller e Friesen (1984) os estágios do ciclo de vida são: (i) Nascimento, (ii) Crescimento, (iii) Maturidade, (iv) Renovação e (v) Declínio.

A utilização do SCG e o uso de informações de natureza gerencial são distintas em cada fase do ciclo de vida das organizações (Su et al., 2015). Com o cenário mercadológico atual, é necessário o desenvolvimento de controles e ferramentas gerenciais, aderentes às necessidades das empresas familiares, observando que uso de informações gerenciais são diferentes em cada etapa do ciclo de vida dessas organizações (Nisiyama \& Oyadomari, 2012). As alavancas de controle gerenciais propostas por Simons (1994) possibilitam realizar a interface com todos os sistemas de gestão de uma empresa, bem como são aderentes ao contexto das empresas familiares, conforme indicam (Moreira \& Frezatti, 2019).

Neste contexto, considerando que a demanda de informações de apoio ao processo decisório é maior e se distingue em cada etapa do ciclo de vida das organizações, o estudo tem por problemática de pesquisa: Como ocorre a interface do sistema de controle gerencial com os estágios do ciclo de vida organizacional de uma empresa familiar? Com o objetivo analisar como ocorre a interface do uso sistema de controle gerencial e alavancas de controle com o ciclo de vida organizacional de empresa familiar.

Como justificativa do estudo, busca-se entender como o sistema de controle gerencial e as transições das fases do ciclo de vida organizacional de uma indústria de alimentos de grande porte, ponderando inclusive que a empresa se encontra em processo de sucessão geracional. Silvola (2008) destaca a importância de estudos de casos, que possam contribuir de maneira mais profundada na análise da relação entre o uso de controles gerenciais e as transições dos estágios do ciclo de vida organizacional. Moreira e Frezatti (2009), também indicam a importância de estudos de casos incluindo empresas familiares.

Quanto às contribuições do estudo, a pesquisa corrobora com a evidenciação das mudanças que ocorrem nas organizações, e a percepção da relação entre a 
Análise dos Sistemas de Controles Gerenciais e a Interface com o Ciclo de Vida de uma Indústria

de Alimentos

Willyam Carlos Pederssetti, Silvana Dalmutt Kruger

utilização de informações e do sistema de controle gerencial, com os estágios do ciclo de vida organizacional, demonstrando a importância das informações gerenciais no processo decisório, especialmente para a continuidade dos negócios familiares.

\section{REVISÃO DA LITERATURA}

Nesta seção apresenta-se o contexto do ciclo de vida das organizações e especificidades da gestão das empresas familiares, bem como a importância da utilização dos sistemas de controles gerenciais.

\subsection{Empresas familiares e o uso de sistemas de controles gerenciais}

O conceito de empresa familiar é entendido como uma organização em que o grupo familiar detém na sua totalidade, ou controla a propriedade desse negócio, e estão diretamente envolvidos membros da família (Pimentel, 2014). Moreira e Frezatti (2019) indicam como características a influência da família na direção estratégica da empresa e o desejo de se sustentar, preservar seus valores entre gerações e manter 0 envolvimento da família no negócio.

A administração familiar torna as empresas mais sensíveis às ameaças ao capital, o qual depende da manutenção do controle familiar e da forma que as metas não econômicas da família são alcançadas (Cennamo, Berrone, Cruz, \& Gomez-Mejia, 2012). Já o capital socioemocional dessas empresas incluem aspectos como: necessidades de pertencimento, afeto e intimidade; continuidade dos valores da família por meio da empresa; perpetuação da dinastia familiar; status social; preservação do capital social da família; quitação de obrigações familiares baseadas em laços de sangue e capacidade de agir de forma altruísta com os membros da família usando recursos da empresa (Mitchell, Agle, Chrisman, \& Spence, 2011).

Diversas razões tornam as empresas familiares distintas das demais, tais como os padrões de propriedade, estrutura de governança, forma de tomada de decisão e as estratégias empresariais adotadas (Pimentel, 2018). Kammerland e Essen (2017) 
Análise dos Sistemas de Controles Gerenciais e a Interface com o Ciclo de Vida de uma Indústria

de Alimentos

Willyam Carlos Pederssetti, Silvana Dalmutt Kruger

afirmam que de certa forma essas empresas são conhecidas pela baixa propensão ao risco, geralmente são conservadoras e estagnadas.

Os gestores responsáveis pelas decisões nas empresas familiares influenciam de forma significativa na estrutura empresarial familiar (Segura \& Formigoni, 2014). Vinagre, Silva, Portugal, Souza, Gonçalves e Pereira (2017) destacam que a filosofia da vida familiar influencia significativamente no estilo da gestão. Segura e Formigoni (2014) complementam que uma das características de gestão das empresas familiares é a baixa aversão ao risco, maior tempo para o processo de tomada de decisão e rotinas estabelecidas.

A sobreposição dos valores familiares com os valores da empresa é um aspecto cultural específico das empresas familiares (Songini et al., 2013). Quando a organização possui executivos contratados e executivos que fazem parte da família, conflitos de interesses podem ocasionar dificuldades na gestão, nesse aspecto o SCG pode contribuir com o planejamento e estabilidade da gestão (Moreira \& Frezatti, 2019). Assim como os SCG podem auxiliar no processo de profissionalização da gestão e no desenvolvimento de instrumentos de apoio ao processo de sucessão familiar (Dekker, Lybaert, Steijvers, Benoît, \& Mercken, 2012).

\subsection{Utilização dos sistemas de controles gerenciais}

Sistemas de Controle Gerencial (SCG) são definidos por Simons (1994) como sistemas de rotinas formais que se baseiam em informações e procedimentos que os gestores utilizam para manter ou alterar as atividades das organizações.

Em seu conceito inicial, os SCGs são caracterizados como procedimentos altamente formalizados que se utilizam de dados para manter ou modificar procedimentos em uma atividade de gestão (Silva \& Beuren, 2020). Essa definição dos SCGs incluem ferramentas de planejamento, modelos de relatórios e processos de monitoramento com a utilização de dados, da mesma forma que o orçamento empresarial, BSC, planejamento estratégico e sistemas de avaliação de desempenho (Della Giustina, Gasparetto, \& João Lunkes, 2020). 
Os SCGs vão muito além do que uma ferramenta de apoio às decisões, pois oferecem um amplo espectro de informações com mecanismos os que garantem que 0 comportamento e decisões dos colaboradores serão aderentes aos objetivos e estratégias das empresas (Truant, Broccardo, \& Vanessa, 2019). Inúmeros objetivos têm sido atribuídos aos SCGs, garantindo que sua utilização pode auxiliar na orientação das organizações, a fim de buscar níveis organizacionais mais desenvolvidos, como os de relação com o cumprimento dos objetivos estratégicos, e inovação de produtos e serviços (Oro \& Lavarda, 2019).

De acordo com Beuren e Oro (2014) os SCGs são um conjunto de ferramentas que os gestores utilizam para manterem as organizações no alcance de seus objetivos. Por meio do emprego de métodos, técnicas, procedimentos e conhecimentos, visam proporcionar o cumprimento das metas organizacionais fixadas. O SCG vinculados às constantes mudanças presentes nas organizações, principalmente familiares, proporcionam importantes modelos para o processo de planejamento e busca dos objetivos de gestão (Moreira \& Frezatti, 2019). Entre as demais empresas, somente há a diferença de que esses sistemas são trabalhados pelos gestores contratados fora da família e os gestores que fazem parte da família.

Simons (1994) defende o conceito de que os SCGs são baseados em quatro alavancas de controle, as quais operam como forças opostas que asseguram o efetivo controle e implementação, sendo compostas: a) Sistema de Crenças, b) Sistemas de Controles Interativos, c) Sistema de Limite e d) Sistemas de Controles Diagnósticos. Esses sistemas devem manter o foco no desempenho das organizações apresentando utilidade e relevância, integrando as funções de planejamento e controle das empresas (Akroyd \& Kober, 2020).

Simons (1994) considera que as alavancas de controle estão fundamentadas na crença de que as empresas que se utilizam delas podem prosperar de forma diferenciada, alinhando adequadamente suas características internas a um cenário competitivo de mercado. Dentre as ferramentas de controle existem as ferramentas de controles contábeis, os quais protegem patrimônios e informações relacionadas à toda 
contabilidade da organização. Por sua vez, as ferramentas de controles administrativos englobam o planejamento e as estratégias que buscam garantir o aumento da eficiência da organização (Sgrò, Palazzi, Ciambotti, \& Gelsomini, 2020). Essas ferramentas são caracterizadas como alavancas de controle interno nesses dois cenários.

As alavancas de controle proporcionam quatro diferentes formas de aplicação dentro dos SCGs, assim, os decisores precisam aplicá-las de forma que seu uso seja integrado e balanceado quando for colocada a estratégia em prática, para assim poder monitorá-la (Cruz, Frezatti, \& Bido, 2015). Uma estrutura formada por um quadro de alavancas proporciona o aumento do controle de maneira estratégica das empresas, todavia a maneira como os decisores utilizam esses sistemas é de grande valia para as organizações (Sgrò et al., 2020).

O estudo de Oro e Lavarda (2019) indicam que os valores culturais da gestão familiar se manifestam nos sistemas de crenças que moldam o uso dos sistemas de controles gerenciais, refletindo no comportamento estratégico da organização.

Assim também como indicado por Moreira e Frezatti, (2019), a utilização das alavancas de controle possibilitam realizar uma interface com todos os sistemas de gestão de uma empresa, as alavancas idealizadas por Simons (1994), são aderentes ao contexto das empresas familiares.

A sobreposição dos valores familiares com os valores da empresa é um aspecto cultural específico das empresas familiares, logo as Alavancas de Controle propostas por Simons (1994), demonstram-se aderentes à realidade das empresas familiares pelo apoio ao desenvolvimento de estratégias que possibilitam o desenvolvimento de iniciativas de melhorias. O uso relacionado das Alavancas de Controle com os sistemas de controle desempenha um papel de desenvolvedor de diagnósticos para assegurar que as estratégias sejam robustas e aplicáveis as distintas realidades empresariais (Nisiyama \& Oyadomari, 2012). 
Análise dos Sistemas de Controles Gerenciais e a Interface com o Ciclo de Vida de uma Indústria de Alimentos Willyam Carlos Pederssetti, Silvana Dalmutt Kruger

\subsection{Ciclo de vida das organizações}

Um dos estudos seminais na abordagem do ciclo de vida organizacional, Chandler (1962) buscou compreender as etapas de desenvolvimento das organizações. Atualmente o conceito do ciclo de vida organizacional tem sido amplamente utilizado nas ciências sociais por sua adaptação às questões referentes ao desenvolvimento e ao crescimento das organizações (Carvalho et al., 2010).

A função da análise do ciclo de vida é de investigar as restrições, incentivos e estratégias durante toda trajetória da organização. Ademais como essas se relacionam com as decisões e resultados de desempenho (Drake, 2012). O surgimento do conceito de ciclo de vida organizacional está intimamente relacionado à teoria biológica, pois considera a trajetória evolucional da empresa, que vai do seu nascimento, passa pela fase de crescimento e, eventualmente, pode chegar à morte (Moreira \& Frezatti, 2019).

Existem diversos modelos de estágios de ciclo de vida das organizações, todavia o apresentado por Miller e Friesen (1984) é o que mais aprofunda seus conceitos e agrega estudos empíricos relacionados (Necyk \& Frezatti, 2010). O modelo de Miller e Friesen (1984) apresenta os estágios evolutivos em cinco fases, sendo elas: Nascimento, Crescimento, Maturidade, Renovação e Declínio, conforme apresenta-se no Quadro 1: 


\begin{tabular}{|c|c|c|}
\hline ão & a & dec \\
\hline $\begin{array}{l}\text { Nascimento: A empresa tem } \\
\text { até } 10 \text { anos. Novas e } \\
\text { pequenas em relação aos } \\
\text { concorrentes. Concentração } \\
\text { de poder e decisão nos } \\
\text { proprietários. }\end{array}$ & $\begin{array}{l}\text { Simples e centralizada. Dirigida } \\
\text { pelos donos. Atividades } \\
\text { administrativas simples com } \\
\text { poucos controles formais ou } \\
\text { uso mínimo de sistemas de } \\
\text { informações. }\end{array}$ & $\begin{array}{l}\text { Busca viabilidade do negócio. } \\
\text { Proatividade no desenvolvimento de } \\
\text { produtos e serviços. Geração de } \\
\text { competências. Riscos substanciais. } \\
\text { Decisões baseadas em intuição, sem } \\
\text { análise detalhada de projetos ou } \\
\text { consideração de alternativas. }\end{array}$ \\
\hline $\begin{array}{l}\text { Crescimento: Empresa já } \\
\text { tem estabilidade no mercado } \\
\text { e demonstra potencialidade } \\
\text { do negócio. Maiores que na } \\
\text { fase anterior. Maior } \\
\text { heterogeneidade do ambiente } \\
\text { externo. }\end{array}$ & $\begin{array}{l}\text { a funcional } \\
\text { mental. Mais complexa } \\
\text { entralizada que na fase } \\
\text { Maior formalização e } \\
\text { amentor de } \\
\text { ções, monitoramento e } \\
\end{array}$ & $\begin{array}{l}\text { Busca crescimento com diversificação } \\
\text { e inovação de produtos. Tomada de } \\
\text { decisões mais analíticas e integradas, } \\
\text { racionais e baseadas em } \\
\text { informações, como por exemplo, } \\
\text { performance financeira dos produtos. }\end{array}$ \\
\hline $\begin{array}{l}\text { Maturidade: Maiores e mais } \\
\text { velhas que nas fases } \\
\text { anteriores. Possuem } \\
\text { vantagem competitiva sobre } \\
\text { os concorrentes. Menor grau } \\
\text { de incerteza do ambiente } \\
\text { externo. }\end{array}$ & 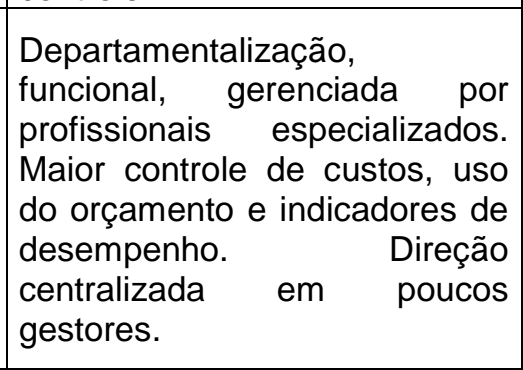 & $\begin{array}{l}\text { Maior aversão ao risco, mais } \\
\text { conservadora, foco em controle de } \\
\text { custos, e margens de lucros. Tomada } \\
\text { de decisão nas mãos de poucos } \\
\text { diretores devido à estabilidade e } \\
\text { simplicidade das operações. No } \\
\text { entanto, há maior peso na análise de } \\
\text { alternativas, procura pela } \\
\text { produtividade e eficiência. }\end{array}$ \\
\hline $\begin{array}{l}\text { nas } \\
\text { inte } \\
\text { ico } \\
\text { dos } \\
\text { ros }\end{array}$ & 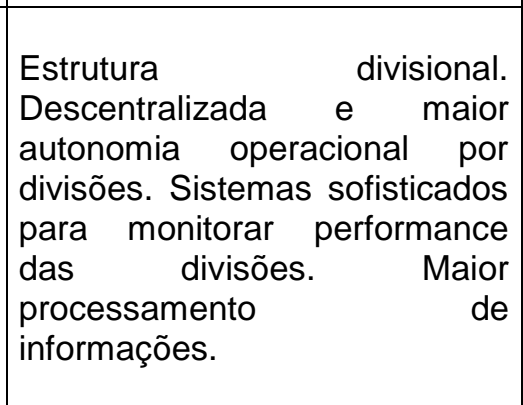 & $\begin{array}{l}\text { Maior foco por inovação, } \\
\text { diversificação e expansão de produtos } \\
\text { e mercados, com maiores riscos, } \\
\text { revertendo a inércia da Maturidade. } \\
\text { Tomada de decisões de forma } \\
\text { reflexiva, analítica e participativa, } \\
\text { devido a maior propensão a risco e } \\
\text { análise do ambiente (oportunidades e } \\
\text { ameaças). Planejamento e foco na } \\
\text { evolução futura da empresa. }\end{array}$ \\
\hline $\begin{array}{l}\text { Declínio: Estagnação, } \\
\text { estratégia defi } \\
\text { Necessidades dos dc } \\
\text { acima da dos clie } \\
\text { Ambiente hostil. }\end{array}$ & \begin{tabular}{|r|} 
à \\
e \\
les \\
de \\
jes
\end{tabular} & $\begin{array}{l}\text { ora, sem uso das } \\
\text { e análises para tomada } \\
\text { es, foco no curto prazo. } \\
\text { anejamento estratégico. }\end{array}$ \\
\hline
\end{tabular}

\section{Quadro 1. Modelo de Ciclo de Vida Organizacional}

Fonte: Adaptado de Moreira e Frezatti (2019) e Miller e Friesen (1984).

Moreira e Frezatti (2019) buscaram compreender o papel do sistema de controle gerencial na evolução dos estágios do ciclo de vida organizacional, no que tange às transições entre estágios em uma empresa familiar brasileira. Os achados da pesquisa 
Análise dos Sistemas de Controles Gerenciais e a Interface com o Ciclo de Vida de uma Indústria

de Alimentos

Willyam Carlos Pederssetti, Silvana Dalmutt Kruger

apontam para a necessidade de formalização de controles gerenciais conforme a empresa passa para os estágios mais avançados do ciclo de vida organizacional.

\section{MATERIAIS E MÉTODOS}

A presente pesquisa classifica-se quanto aos seus objetivos como descritiva, quanto aos procedimentos caracteriza-se como um estudo de caso com análise qualitativa, realizado por meio de entrevistas com gestores, a coleta de dados ocorreu com o auxílio de um roteiro estruturado baseado na metodologia adaptada de Miller e Friesen (1984) e Moreira e Frezatti (2019).

A empresa objeto do estudo está sediada em um município de aproximadamente 25.000 habitantes, no Oeste do estado de Santa Catarina, se enquadra como empresa de grande porte, considerando seu faturamento e quantidade de colaboradores, com base na classificação do Sebrae (2020), sendo uma Sociedade Anônima de capital fechado. Sua atividade está voltada ao setor alimentício com fabricação de massas e biscoitos, sua gestão é familiar. A empresa possui aproximadamente 620 colaboradores, divididos nos setores de produção, logística, suprimentos, comercial, recursos humanos, contábil, projetos, tecnologia da informação e financeiro.

A amostra da pesquisa é composta por 8 colaboradores, sendo 6 gestores e 2 diretores, a estrutura da empresa contempla os seguintes departamentos: Direção, Recursos Humanos, Produção, Pesquisa e Desenvolvimento, Comercial, Financeiro e Logística, com seus representantes de maiores níveis hierárquicos que possuem relação com a tomada de decisão da empresa em estudo.

As entrevistas com os 8 gestores ocorreram no período de 22 de junho/2020 a 06 de julho/2020. Primeiramente foram agendados os horários pelos pesquisadores com todos os respondentes, os quais foram entrevistados no ambiente da organização, com entrevistas realizadas de forma presencial com um tempo médio de quarenta e oito minutos de duração. Após a realização das 8 entrevistas (sendo 2 com diretores e 6 
Análise dos Sistemas de Controles Gerenciais e a Interface com o Ciclo de Vida de uma Indústria

de Alimentos

Willyam Carlos Pederssetti, Silvana Dalmutt Kruger

com gerentes), as informações foram organizadas e comparadas em uma planilha eletrônica. A caracterização dos respondentes está apresentada na Tabela 1.

Tabela 1

Caracterização dos respondentes e tempos de entrevistas dos colaboradores

\begin{tabular}{lccccc}
\hline \multicolumn{1}{c}{ Cargo } & Idade & Escolaridade & $\begin{array}{c}\text { Tempo de } \\
\text { empresa (anos) }\end{array}$ & $\begin{array}{c}\text { Tempo na } \\
\text { função } \\
\text { (anos) }\end{array}$ & $\begin{array}{c}\text { Tempo de } \\
\text { entrevista } \\
\text { (minutos) }\end{array}$ \\
\hline Diretor Administrativo & 62 & Superior Incompleto & 32 & 32 & 47 \\
Diretor Adjunto & 56 & Pós-Graduado & 20 & 20 & 52 \\
Gerente Comercial & 55 & Superior Completo & 29 & 20 & 62 \\
Gerente Financeiro & 54 & Pós-Graduado & 31 & 30 & 56 \\
Gerente de Logística & 42 & Pós-Graduado & 1 & 6 & 58 \\
Gerente de Produção & 53 & Superior Completo & 2 & 2 & 70 \\
Gerente de RH & 30 & Pós-Graduado & 5 & 1 & 55 \\
Gerente de P\&D & 41 & Pós-Graduado & 14 & 5 & 49 \\
\hline
\end{tabular}

Nota. Fonte: Dados da pesquisa (2020).

A análise dos resultados foi elaborada após a coleta dos dados primários transmitidos para planilhas eletrônicas de apoio. Primeiramente foram tabuladas as questões de cada respondente, bem como com sua caracterização. Posteriormente foram analisadas as informações, tanto para o nível de maturidade e uso de controles gerenciais, quanto para o ciclo de vida organizacional, observando-se as dimensões: Crenças da Empresa, Relação Interpessoal; Monitoramento de Resultados, Compliance, Responsabilidade Social, Inovação e Uso de Controle e Informações. As respostas obtidas foram categorizadas a partir do constructo de Simons (1994), apresentando-se o diagnóstico, baseado nas alavancas de controles gerenciais, relacionadas com as estratégias e oportunidades para o desenvolvimento de iniciativas de melhorias para a empresa estudada, observando as características relacionadas ao ciclo de vida. 
Análise dos Sistemas de Controles Gerenciais e a Interface com o Ciclo de Vida de uma Indústria

de Alimentos

Willyam Carlos Pederssetti, Silvana Dalmutt Kruger

\section{RESULTADOS}

Atualmente, a empresa ambiente do estudo, tem sua constituição societária caracterizada por uma sociedade anônima de capital fechado, com gestão exclusivamente familiar, possui a inclusão de holdings companies na sociedade, o controle do grupo econômico é formado por um conselho de administração e presidido pela família. Os resultados das 8 entrevistas realizadas foram separados em sete conjuntos (blocos) de questionamentos e análise: (i) Crenças da Empresa; (ii) Relação Interpessoal; (iii) Monitoramento de Resultados; (iv) Compliance; (v) Responsabilidade Social; (vi) Inovação; (iv) Uso de Controle e Informações.

(i) No bloco de perguntas com relação as crenças da empresa, a primeira questão foi "Com relação às crenças da companhia, o(a) Senhor(a) acredita que a Missão, Visão e Valores são divulgados para todos os integrantes da organização?" o Diretor administrativo, com mais tempo de função na empresa, respondeu "Apesar de ser divulgado a todos em algumas oportunidades e as ações estejam alinhadas, acredito que efetivamente são assimiladas as diretrizes aos colaboradores com cargos de liderança" assim também como os demais respondentes indicaram que a divulgação ocorre, sendo melhor absorvida pelas funções de liderança na empresa. Nesse sentido, Moreira e Frezatti (2019) afirmam que a manutenção de um ciclo de vida sustentável em uma empresa familiar está relacionada com a formalização das diretrizes gerenciais e do seu entendimento por parte dos diversos níveis colaborativos.

Ao serem questionados " $O(A)$ Senhor(a) acredita que a Missão, Visão e Valores da empresa são claros?" todos os respondentes classificaram como claras e compreensíveis as diretrizes da empresa, com exceção de um respondente, o qual indicou que a informação pode chegar distorcida ao destino, pelas características da empresa ser de gestão familiar. Outro aspecto observado nesta resposta, é o menor tempo de empresa do respondente, que pode não ter internalizado a cultura empresarial familiar. 
Análise dos Sistemas de Controles Gerenciais e a Interface com o Ciclo de Vida de uma Indústria

de Alimentos

Willyam Carlos Pederssetti, Silvana Dalmutt Kruger

$\mathrm{Na}$ terceira questão deste bloco, verificou-se "Essas crenças compartilhadas descrevem os valores fundamentais defendidos pela organização?" seis respondentes indicam que sim, elas descrevem em sua totalidade, já dois respondentes divergem, indicando que as crenças não descrevem em sua totalidade os valores da empresa. Logo essa discordância com as diretrizes gerenciais, aplicadas à crença e os valores da empresa, assim também como verificado no estudo de Moreira e Frezatti (2019) pode elevar a gerência de uma empresa familiar a uma gestão mais participativa e inclusiva.

No último questionamento do bloco "crenças da empresa", perguntou-se "Essas crenças da organização servem como pano de fundo (base) para a definição de estratégias e norteiam a organização como um todo?". As respostas indicam quase em sua totalidade que essas crenças são a base para as definições dos investimentos, desenvolvimentos de novos produtos e processos. Apenas uma resposta indicou que essas crenças não são utilizadas em sua totalidade para definição das estratégias da empresa.

Assim como definem Wang, Lo e Weng (2019), uma empresa familiar com crenças muito bem definidas e transmitidas para seus stakeholders facilita o processo de inovação sustentável, apoiando inclusive o processo de sucessão familiar. Logo, a empresa pesquisada deverá criar mecanismos para solidificar e difundir de forma ampla suas crenças e valores, em todos os níveis corporativos, visando corrigir a fragilidade percebida quanto ao alinhamento das crenças e valores organizacionais.

(ii) No bloco de questões voltados às relações interpessoais, perguntou-se "Os superiores, frequentemente, envolvem os subordinados em atividades de gestão ou convidam para ajudar na tomada de decisões importantes?" Os respondentes divergiram com relação às respostas. A direção indica que deve ser uma prática comum o envolvimento dos subordinados nas decisões, porém reconhecem que alguns gestores têm perfil mais participativos que outros. Já alguns gestores afirmam que existem pontos falhos, principalmente de comunicação.

No segundo questionamento, observou-se "Existe formalmente um momento ou maneira para que os servidores colaborem com opiniões sobre oportunidades para a 
Análise dos Sistemas de Controles Gerenciais e a Interface com o Ciclo de Vida de uma Indústria

de Alimentos

Willyam Carlos Pederssetti, Silvana Dalmutt Kruger

organização?", novamente as respostas são divergentes, parte da direção indica haver uma maneira formal, em reuniões de gestores e do conselho de administração, parte da direção indica não haver uma maneira formal de comunicação para sugestões. Entre os gestores também se constatou essa divergência, alguns indicam a existência de um canal formal de colaboração de opiniões e outros desconhecerem esse meio.

Nesse sentido, Oro e Lavarda (2019) indicaram que um canal formal de comunicação entre todos os níveis hierárquicos da empresa pode facilitar no processo de inovação de produtos e serviços internos, além de possibilitar o desenvolvimento e as melhorias dos SCGs.

No último questionamento deste bloco, evidenciou-se que "Os gestores procuram estimular o aprendizado organizacional por meio do compartilhamento de experiências e percepções entre os colaboradores?", logo por unanimidade, os respondentes indicaram que as informações estratégicas passam a fazer parte da rotina de atividades dos gestores, os quais difundem à suas equipes. Segundo resposta de um dos diretores, "vivemos em nossa empresa o melhor momento de integração e compartilhamento de informações entre gestores e setores", demonstrando, que a integração no mesmo nível hierárquico, ou seja, gestores, está alinhado com as diretrizes estratégicas.

As respostas deste bloco corroboram com os achados de Raupp, Martins e Beuren (2006), evidenciando que a comunicação entre direção e os colaboradores com cargos de comando das empresas, são elementos fundamentais para a utilização mais eficiente das ferramentas de controle e gestão. Todavia, percebeu-se que a empresa necessita melhorar o canal de comunicação com os colaboradores de todos os níveis hierárquicos, promovendo um debate mais alinhado com as diretrizes estratégicas.

(iii) O bloco monitoramento de resultados possui quatro questões voltadas aos resultados da empresa. Na primeira questão, perguntou-se "A organização possui sistemas de monitoramento de resultados, ou seja, os gestores monitoram, efetiva e periodicamente, os resultados atingidos?". Nesse quesito, a direção e os gestores estão alinhados quanto à resposta, pois todos indicaram que a empresa possui um sistema 
de monitoramento de resultados e que os gestores dispõem das informações mais estratégicas para tomadas de decisões. E como iniciativa de melhoria contínua no processo, seguidamente são incorporados ajustes no sentido de agilizar a entrega das informações em tempo cada vez mais adequado aos interessados.

A segunda questão verificou-se "A companhia tem objetivos e metas claras? Existe monitoramento efetivo e periódico para verificar seu atingimento?", novamente houve indicação pelos respondentes da existência destas metas e objetivos, além de mecanismos de verificação. Essas metas estão relacionadas a fatores mais quantitativos, como meta de vendas, meta de orçamento e resultados, com periodicidade diária, semanal e mensal.

Apenas um dos respondentes indicou que se perdeu a cultura da utilização de indicadores com o passar dos anos na empresa, indicando que os objetivos e metas não são claros para todos os setores, necessitando de melhorias para equalizar a difusão dos objetivos e metas aos gestores. Esses resultados vão ao encontro dos achados de Della Giustina et al. (2020), demonstrando que o alinhamento estratégico da empresa está debruçado na dependência de sistemas e ferramentas robustas de monitoramento de resultados, bem como formar como as informações são transferidas aos usuários, corroborando com a melhoria no ciclo de vida das organizações. Os resultados do presente estudo refletem a importância do alinhamento dos objetivos estratégicos e das metas, fortalecendo a relação entre a clareza dos objetivos estratégicos com os resultados alcançados.

Questionou-se na sequência, "A organização possui planejamento formalmente divulgado?", identificando-se que o planejamento estratégico é compartilhado entre conselho de administração, gestores e líderes, sendo que esses traduzem as suas equipes parâmetros dos quais eles possam contribuir para o cumprimento do planejamento.

E como última questão deste bloco, verificou-se "Em casos de não atendimento pleno da meta, a organização penaliza os colaboradores de alguma forma?". Identificou-se o entendimento de todos respondentes que a empresa possui um sistema 
Análise dos Sistemas de Controles Gerenciais e a Interface com o Ciclo de Vida de uma Indústria de Alimentos Willyam Carlos Pederssetti, Silvana Dalmutt Kruger

global de participação nos resultados. O colaborador deixa de receber se os resultados planejados não foram alcançados. Esses resultados corroboram com estudo de Necyk e Frezatti (2010), os quais afirmam que quanto mais desenvolvido está a fase do ciclo de vida da empresa, maior é a utilização dos SCGs vinculados ao monitoramento dos resultados da empresa.

(iv) Neste quarto bloco de questões, denominado de Compliance, primeiramente questionou-se "A organização possui um código de ética e de conduta? Esse material é difundido entre os colaboradores?", os respondentes indicam que as diretrizes estratégicas contemplam a essência desse código de ética e conduta, porém não há uma formalização desse documento para os colaboradores. Essa deficiência pode gerar um entendimento equivocado de algumas condutas em diferentes setores da empresa.

A segunda questão verificou-se "Os limites quanto à liberdade/autonomia são formalmente declarados aos colaboradores, mesmo em caso de não haver um código de ética explícito?". As respostas indicaram o entendimento (unânime) que a empresa utiliza protocolos e utiliza o setor de Recursos Humanos para mediar e esclarecer atribuições éticas e de conduta no ambiente de trabalho.

Verificou-se também se "A companhia realiza, efetivamente, a punição dos que transgridam as regras da organização, mesmo em caso de não haver um código de ética explícito?". Os respondentes indicam que há punição para esses casos. A empresa dispõe de um comitê de avaliação de conduta não adequada, já que esse comitê avalia a gravidade e penalidade, pois a conduta está vinculada na participação dos resultados da empresa.

Observa-se que a empresa necessita de um processo de formalização de códigos de normas e condutas, visando alinhar a conformidade dos valores institucionais e solidificar a cultura empresarial. Oro e Lavarda (2019) evidenciam que a formalização de normas de conduta auxiliam no alinhamento das condutas profissionais, contribuindo com a gestão de empresas familiares. 
Análise dos Sistemas de Controles Gerenciais e a Interface com o Ciclo de Vida de uma Indústria de Alimentos Willyam Carlos Pederssetti, Silvana Dalmutt Kruger

(v) O quinto bloco, Responsabilidade social, questionou "Quando alguém critica a organização, como o(a) Senhor(a) se sente? Por quê?". Para essa questão todos os respondentes sentem-se incomodados com possíveis críticas por fazerem parte da empresa. Porém, por unanimidade, essas críticas são analisadas posteriormente e utilizadas como iniciativas de melhorias para os pontos indicados.

A segunda questão deste bloco, perguntou se "A imagem que a comunidade/sociedade tem da organização, importa para o(a) senhor(a)? Por quê?". Por unanimidade as respostas indicaram que a imagem é um elemento importante frente à sociedade, que a empresa consolidou sua imagem ao longo de sua história. Esses achados corroboram com os resultados de Javed, Rashid, Hussain e Ali (2020), os quais verificam que a reputação da empresa está intimamente relacionada com a sua imagem e as iniciativas sociais, frente à comunidade a qual está inserida.

O questionamento "O(A) Senhor(a) acredita que a frase: o sucesso desta empresa é o meu sucesso, se aplica a maioria dos colaboradores?" busca verificar a integração pessoal dos gestores com a empresa. Nesse quesito há uma dubiedade na visão dos gestores, quatro respondentes acreditam que muitos colaboradores não dão importância a esse aspecto, pois não há uma ligação afetiva com a empresa a qual trabalham. Três respondentes indicaram que esse é um sentimento da maioria dos gestores e colaboradores da empresa, demonstrando comprometimento e afetividade, indicando uma característica de maturidade no ciclo de vida da empresa.

No quarto quesito deste bloco, perguntou-se "Como o(a) Senhor(a) percebe que os colaboradores se referem à organização quando se remetem a ela. Tratam-na como "nossa empresa" ou "empresa deles"?". Como a anterior, essa questão busca verificar a aderência da integração pessoal dos colaboradores com a empresa. Verificou-se que a maioria das respostas indicaram que os colaboradores tratam como "nossa empresa", demonstrando uma ligação entre os colaboradores com as características familiares da empresa.

Com relação ao desempenho pessoal, perguntou-se "O(A) Senhor(a) acredita que a empresa inspira as pessoas a se doar ao máximo no desempenho de suas 
Análise dos Sistemas de Controles Gerenciais e a Interface com o Ciclo de Vida de uma Indústria

de Alimentos

Willyam Carlos Pederssetti, Silvana Dalmutt Kruger

funções?". Nessa questão os respondentes indicaram que a empresa inspira os colaboradores, que ocorre doação e a busca pelos objetivos organizacionais. Uma característica indicada é que a empresa possui um número elevado de colaboradores com experiência e tempo de atuação na empresa.

A última questão deste bloco solicitou a cada respondente se o mesmo "Acredita que as pessoas estão verdadeiramente preocupadas com o futuro da organização?". Seis dos respondentes indicaram que os colaboradores têm essa preocupação. Dois dos respondentes entendem que a maioria dos colaboradores não possuem essa preocupação com a continuidade do negócio. De acordo com as respostas deste bloco denominado "Responsabilidade social", os sistemas de controles gerenciais necessitam de melhorias na avaliação da aceitação da empresa frente à comunidade, podendo ser potencializadas mudanças na imagem e na fase do seu ciclo de vida.

(vi) Este bloco de perguntas denominado inovação, busca verificar em qual nível a empresa está operando suas iniciativas de inovação. A primeira questão indagou "Como se dá o processo de criação de novos produtos na organização?". As respostas revelaram que há na empresa um grupo de pessoas que compõe um comitê de desenvolvimento de novos produtos, liderados pelo gestor de P\&D e Qualidade. Esse processo se inicia observando as tendências de consumos do mercado regional e nacional. Também há uma avaliação dos limites e barreiras impostas pelos fornecedores e linhas de produção, por meio de uma pesquisa mais refinada de potencial e preço a ser praticado, tamanho ideal de embalagem, composição final do produto e faixa de consumidores e clientes.

A segunda questão complementa a primeira, ao ser indagado "Existe um setor responsável por criar novidades? Há algum tipo de meta de produtividade anual/mensal? Quais os parâmetros adotados para lançamento de um novo produto no mercado?". Os respondentes indicaram que há um setor específico responsável por desenvolvimento de novos produtos e há metas para o lançamento desses. Porém, observa-se na empresa que tão importante quanto o lançamento diversos produtos no mercado, é manter a assertividade do lançamento de algo realmente diferenciado e que 
agregue valor, com margem atrativa para a empresa. Os parâmetros indicados pelos respondentes são simples: ser altamente rentável e vendável.

Beuren e Oro (2014) indicam que o processo de inovação de produtos nas indústrias desempenha um papel relevante no desenvolvimento da empresa, juntamente com a utilização dos SCGs. Oro e Lavarda (2019) explanam que um processo formal estruturado de inovação em uma indústria de gestão familiar possui um papel relevante na diferenciação competitiva frente ao mercado. Simons (1994) evidencia que os parâmetros de rentabilidade e de venda das inovações geradas pelas empresas, são importantes para traçar o planejamento estratégico e o auxílio no desenvolvimento dos SCGs.

Ainda no âmbito dos processos inovadores, a terceira questão do bloco "inovação" buscou identificar "Com relação à processos inovadores, os colaboradores são chamados a contribuir com ideias e sugestões? Como funciona esse processo?". A pesquisa identificou que a maioria das sugestões surge de problemas existentes, mas não há na empresa um canal exclusivo para esse fim. Quando há identificação de novos problemas, as pessoas envolvidas são chamadas para contribuir. Como prática recorrente, os gestores salientam que normalmente ocorrem reuniões setoriais para analisar processos que apresentem problemas de entrega. Nessas reuniões com os setores envolvidos são analisados os problemas e as possíveis soluções, impactos e demandas, para então aprovar o processo de mudança ou inovação.

O último questionamento deste bloco, procurou identificar se "Existe algum incentivo, premiação ou concurso para os colaboradores participarem das inovações da organização?". A empresa possui uma política de participação nos lucros com regras e comitê julgador próprio. Porém não existe premiação individual para incentivar sugestões de inovações. Um dos motivos indicados para não haver esse processo na empresa é a alta integração dos processos entre os setores. Como sugestão de desenvolvimento de uma cultura de inovação na empresa, corroboram com os achados o estudo de Beuren e Oro (2014), indicando que é necessário o envolvimento dos 
Análise dos Sistemas de Controles Gerenciais e a Interface com o Ciclo de Vida de uma Indústria de Alimentos Willyam Carlos Pederssetti, Silvana Dalmutt Kruger

colaboradores no processo inovativo, bem como o alinhamento com os sistemas de controles gerenciais.

(vii) Neste último bloco de questões, é indagado aos respondentes sobre a utilização de controles e informações. A primeira questão buscou identificar "A empresa faz uso de controles/ferramentas gerenciais? Quais? E com qual periodicidade?". As respostas demonstram que a alta gestão utiliza como principal ferramenta a análise de resultado e indicadores específicos. Mensalmente há uma validação do resultado pelo departamento de controladoria, e semanalmente é analisado o desempenho parcial, e nesse momento são traçadas estratégias de curto prazo para alcance dos objetivos que estão desalinhados com a estratégia de longo prazo.

Complementando a primeira questão, indagou-se aos respondentes "De qual(is) ferramenta(s) tecnológica(s) a empresa faz uso para a sua gestão?”. Identificou-se que o sistema ERP (Enterprise Resource Planning) utilizado é reconhecido a nível mundial, o qual é interligado a praticamente todos os demais softwares específicos para o Departamento Pessoal, Comercial e Logística. Na produção são utilizados sistemas próprios para o monitoramento de desempenho fabril, avaliando os custos de produção, bem como o volume e a eficiência, os quais também são interligados ao sistema principal.

Na terceira questão verificou-se "A empresa dispõe de planejamento estratégico? Com que periodicidade o documento é revisado?". As respostas indicaram que a empresa conta com planejamento estratégico com diretrizes aprovadas pelo conselho de administração. O ciclo de revisão é anual com projeção do planejamento para cinco anos. Os resultados encontrados nesse quesito da pesquisa estão alinhados aos achados de Akroyd e Kober (2020), os quais indicam que a utilização dos SCGs deve corroborar com o desenvolvimento e acompanhamento do planejamento estratégico.

Muito semelhante às questões relacionadas com a inovação, perguntou-se, "Existe um plano ou cronograma para lançamento de novos produtos?". As respostas indicaram que no passado existiu uma meta de lançamento de novos produtos, enquanto havia disponibilidade nas linhas de produção. Com a utilização da capacidade 
Análise dos Sistemas de Controles Gerenciais e a Interface com o Ciclo de Vida de uma Indústria de Alimentos Willyam Carlos Pederssetti, Silvana Dalmutt Kruger

das linhas o desenvolvimento de novos produtos é atrelado à definição de investimentos em novas linhas que permitam produzir uma nova gama de produtos, logo há necessidade de investimentos em inovação de processos para 0 desenvolvimento de novos produtos.

A última questão procurou evidenciar "Como é composta a estrutura de gestão da companhia?". Os respondentes indicaram que a estrutura é composta por uma alta gestão na companhia, a qual é exercida conjuntamente entre: Assembleia, Conselho Administrativo, Diretoria e Gerência.

A Figura 1 ilustra de forma resumida o arcabouço dos blocos com os principais resultados encontrados, acerca das alavancas de controles gerenciais da empresa analisada. 
Análise dos Sistemas de Controles Gerenciais e a Interface com o Ciclo de Vida de uma Indústria de Alimentos Willyam Carlos Pederssetti, Silvana Dalmutt Kruger

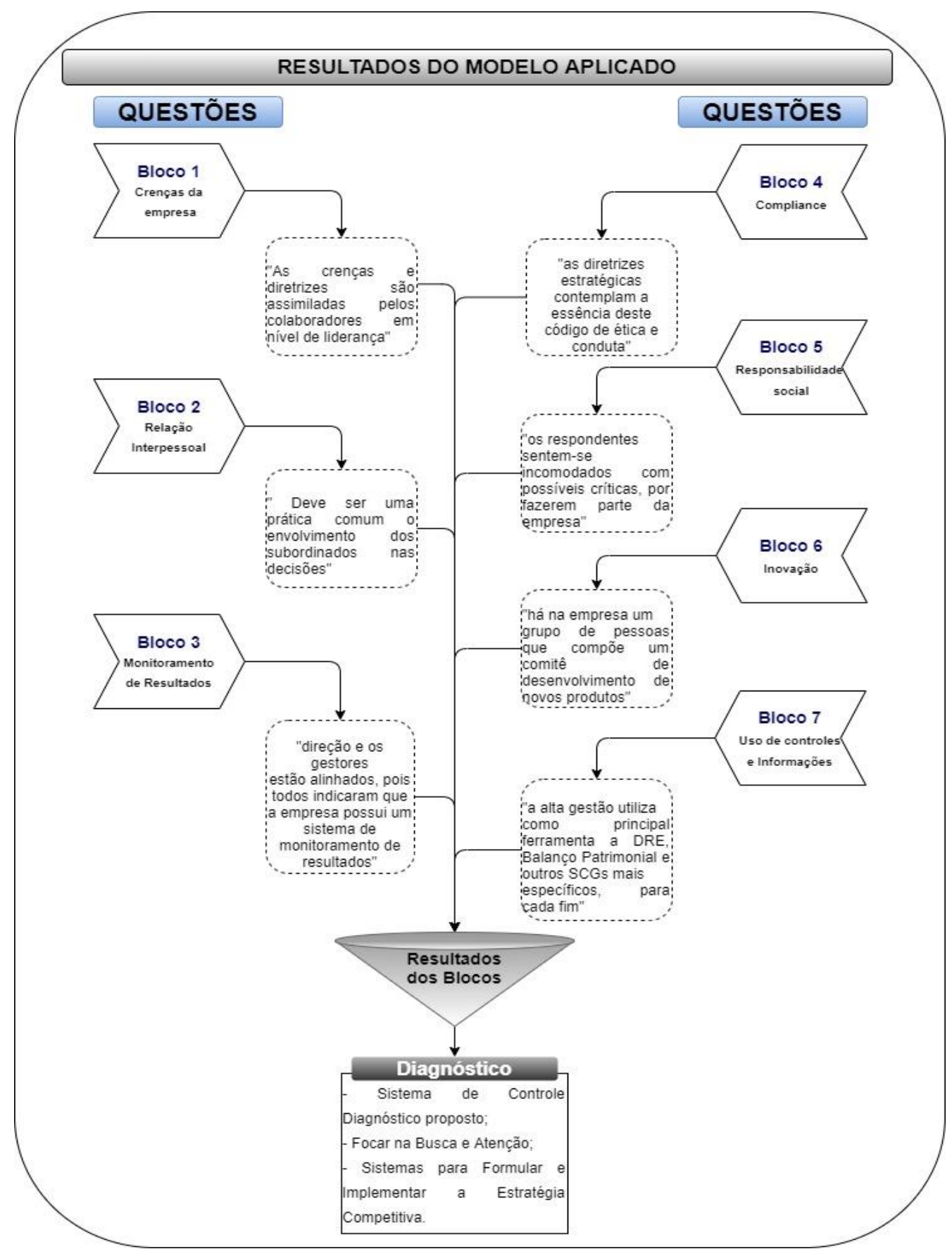

Figura 1. Principais resultados do modelo aplicado

Fonte: Dados da pesquisa (2020).

A Figura 1 demonstra os principais resultados de cada um dos blocos observados no desenvolvimento da pesquisa. No Bloco "Crenças da Empresa", os 
resultados indicam que as crenças e diretrizes são assimiladas pelos colaboradores em nível de liderança, vindo de encontro com os resultados do Bloco "Compliance", o qual indica que as diretrizes estratégicas contemplam a essência do código de ética e conduta assumido pela empresa. O Bloco "Monitoramento de Resultados", o qual sugere que a direção e os gestores estão alinhados, pois todos indicaram que a empresa possui um sistema de monitoramento de resultados, relaciona-se com o Bloco "Uso de Controles e Informações", pela alta gerência utilizar como principal ferramenta de apoio ao processo decisório o Demonstrativo de Resultado, o Balanço Patrimonial e outras informações gerenciais específicas para cada diretoria ou setor.

Quanto às Alavancas de Controle Gerenciais, os resultados viabilizaram a construção de um diagnóstico para posicionar a empresa quanto seu estado atual, sendo demonstrado que no quadrante de Sistema de Controle Diagnóstico, a empresa possui oportunidades no desenvolvimento de Sistemas para Focar Busca e Atenção e estratégia de Sistemas para Formular e Implementar a Estratégia Competitiva. Esse enquadramento traduz a necessidade da empresa desenvolver seus sistemas de gestão, com foco em relatórios voltados ao desempenho financeiro. Outro ponto de destaque é a adequação de treinamentos pessoais para adequar a equipe com relação à atenção na continuidade dos negócios, voltados para o alinhamento coletivo aos objetivos, conforme preconizado por Simons (1994).

Após a análise do SCG foi possível identificar o posicionamento da empresa quanto às fases do seu ciclo de vida, conforme se apresenta no Quadro 2. 
Análise dos Sistemas de Controles Gerenciais e a Interface com o Ciclo de Vida de uma Indústria

de Alimentos

Willyam Carlos Pederssetti, Silvana Dalmutt Kruger

\begin{tabular}{|c|c|c|}
\hline $\begin{array}{l}\text { Fase e } \\
\text { Situação }\end{array}$ & Estrutura/Características & $\begin{array}{l}\text { Estratégias / Estilo de tomada de } \\
\text { decisões }\end{array}$ \\
\hline Nascimento & $\begin{array}{l}\text { A empresa nasceu da cisão de um grupo } \\
\text { já existente na segunda metade da } \\
\text { década de 1980, com administração } \\
\text { familiar e administração centralizada nos } \\
\text { proprietários. }\end{array}$ & $\begin{array}{l}\text { Todas as decisões eram emitidas pelos } \\
\text { sócios administradores da empresa, a } \\
\text { empresa ainda não possuía uma } \\
\text { administração profissional. }\end{array}$ \\
\hline Crescimento & \begin{tabular}{|l} 
A aquisição de novas linhas de produção e \\
o crescimento econômico da primeira \\
metade da década de 1990, \\
impulsionaram a necessidade de \\
crescimento e expansão da empresa.
\end{tabular} & $\begin{array}{l}\text { A empresa procurou analisar melhor o } \\
\text { mercado, e iniciou um plano de expansão } \\
\text { a longo prazo, baseado na } \\
\text { profissionalização da empresa e na } \\
\text { inovação de seus produtos. }\end{array}$ \\
\hline Maturidade & $\begin{array}{l}\text { A atual fase em que a empresa } \\
\text { encontra, com maior nível de } \\
\text { profissionalização, e u utilização de } \\
\text { ferramentas mais consistentes de gestão. } \\
\text { As decisões são baseadas em orçamentos } \\
\text { anuais e gestão de custos produtivos, bem } \\
\text { como análise de margem de contribuição. }\end{array}$ & $\begin{array}{l}\text { A equipe de gestão é composta por seis } \\
\text { gestores e dois diretores, houve } \\
\text { recentemente a implementação do } \\
\text { orçamento anual e implantação de } \\
\text { estratégias baseadas em Balanced } \\
\text { Scorecard. }\end{array}$ \\
\hline Renovação & $\begin{array}{l}\text { A empresa planeja a renovação do quadro } \\
\text { de direção, por meio de um plano de } \\
\text { sucessão. Há também um investimento } \\
\text { maciço em tecnologia da informação e } \\
\text { inovação em processos e produtos. }\end{array}$ & $\begin{array}{l}\text { A realização de um planejamento } \\
\text { sucessório com a consultoria de empresa } \\
\text { especializada é o principal aspecto na } \\
\text { estratégia de renovação e continuidade da } \\
\text { empresa. }\end{array}$ \\
\hline
\end{tabular}

\section{Quadro 2. Percepções do Ciclo de Vida Organizacional}

Fonte: Elaborado pelos autores (2020).

Pode-se identificar que a empresa encontra-se na fase de maturidade em seu ciclo de vida, caracterizado por seu maior nível de profissionalização, e utilização de ferramentas mais consistentes de auxílio a tomada de decisão. A gestão é baseada em orçamentos anuais e análises de custos produtivos, bem como acompanhamento da margem de contribuição. A alta gestão da empresa é composta por seis gestores e dois diretores, compreendendo todos departamentos. Um ponto de destaque recente foi a implementação do orçamento anual e implantação de estratégias baseadas em Balanced Scorecard.

Essa composição na estrutura de gestão baseada em decisão de um grupo familiar também é indicada por Su et al. (2015), os quais indicam que o ciclo de vida organizacional está relacionado diretamente com a estrutura de gestão e a forma de utilização dos SCGs. 
Análise dos Sistemas de Controles Gerenciais e a Interface com o Ciclo de Vida de uma Indústria

de Alimentos

Willyam Carlos Pederssetti, Silvana Dalmutt Kruger

Assim, como de acordo com a classificação do ciclo de vida organizacional de Moreira e Frezatti (2019) a empresa por ter sua gestão familiar, possui maior aversão ao risco, é mais conservadora e tem o foco no controle de custos e margens de lucros. A tomada de decisão está nas mãos de dois diretores. No entanto, há um peso significativo na definição de estratégias capazes de melhorar a eficiência operacional e administrativa.

\section{CONCLUSÃO}

Como principal destaque dos resultados, verificou-se que os dois blocos (Monitoramento dos resultados e Utilização de controles e informações) contribuem para promover a difusão das estratégias, cada uma desempenhando uma função: monitoramento dos resultados proporciona uma visão de curto prazo com perspectiva de médio e longo prazo para o atingimento das metas. Já a utilização de controles e informações, auxiliam diretamente na definição das estratégias corporativas, proporcionando suporte e diretrizes para os diversos stakeholders.

Percebeu-se por meio da pesquisa, que a empresa está posicionada no nível de Maturidade, em seu ciclo de vida organizacional. Indicado pelo tempo de mercado, e pelas vantagens competitivas frente aos concorrentes, além de demonstrar baixo grau de incerteza do ambiente externo. A estrutura de gestão é departamental, em partes complexa e descentralizada, há formalização e processamento de informações, monitoramento e controle, podendo ser melhorada essa última.

Constatou-se que a Inovação é percebida como um aspecto que requer o comprometimento de todos os colaboradores da empresa, principalmente no diálogo necessário entre o grupo de pessoas que compõe o comitê de desenvolvimento de novos produtos. $E$ que existe a preocupação com o alinhamento da inovação com a Responsabilidade Social da empresa, fator esse que é determinante na aceitação das inovações da empresa frente à sociedade. 
Análise dos Sistemas de Controles Gerenciais e a Interface com o Ciclo de Vida de uma Indústria

de Alimentos Willyam Carlos Pederssetti, Silvana Dalmutt Kruger

Os resultados também indicaram que a empresa possui uma gama de ferramentas pautadas nos SCGs, porém muitas vezes são utilizadas pela alta gestão, não sendo difundida para a utilização por outros stakeholders. Visto que um canal formal de comunicação entre todos os níveis hierárquicos da empresa pode facilitar o processo de definição das estratégias empresariais, além de possibilitar o desenvolvimento e difusão dos SCGs.

Quanto às Alavancas de Controle Gerenciais, os resultados viabilizaram a construção de um diagnóstico para posicionar a empresa quanto ao seu estado atual, sendo demonstrado que no quadrante de Sistema de Controle Diagnóstico, a empresa possui oportunidades no desenvolvimento de Sistemas para Focar Busca e Atenção e estratégia de Sistemas para Formular e Implementar a Estratégia Competitiva. Esses resultados possibilitam o desenvolvimento de um plano estratégico de longo prazo por parte da empresa, a fim de mitigar os riscos apresentados e agregar melhorias em seus resultados.

Algumas limitações podem ser observadas nesta pesquisa. A metodologia de coleta de dados foi definida sobre um modelo específico, podendo ter ficado de forma despercebida, sendo que alguns detalhes poderiam contribuir para a complementação de algumas visões adicionais aos gestores. Os níveis hierárquicos de supervisão não foram pesquisados, podendo revelarem situações distintas e visões mais próximas ao ambiente operacional.

Para o desenvolvimento de futuras pesquisas, sugere-se a aplicação de metodologias capazes de proporcionar iniciativas de melhorias na definição de estratégias com base em ferramentas dos SCGs em empresas familiares. Essas iniciativas necessitam estarem pautadas em elementos consistentes da gestão familiar da empresa, e em consonância com os gestores, que muitas vezes não fazem parte da família. 


\section{REFERÊNCIAS}

Akroyd, C., \& Kober, R. (2020). Imprinting founders' blueprints on management control systems. Management Accounting Research, 46(100645).

Bertucci, J. L. O. (2009). Metodologia básica para elaboração de trabalhos de conclusão de cursos (TCC). São Paulo: Atlas.

Beuren, I. M., \& Oro, I. M. (2014). Relação entre estratégia de diferenciação e inovação, e sistemas de controle gerencial. Revista de Administração Contemporânea, 18(3), 287-310.

Carvalho, K. L., Júnior, A. F. A., Frezatti, F. \& Costa, R. P. (2010). A contribuição das teorias do ciclo de vida organizacional para a pesquisa em contabilidade gerencial. Revista de Administração Mackenzie, 11(4), 98-130.

Cennamo, C., Berrone, P., Cruz, C., \& Gomez-Mejia, L. R. (2012). Socioemotional Wealth and Proactive Stakeholder Engagement: Why Family-Controlled Firms Care More About Their Stakeholders. Entrepreneurship Theory and Practice, 36(6), 11531173.

Chandler, A. D. (1962). Strategy and structure: chapters in the history of the industrial enterprises. Cambridge, Mass.: MIT Press.

Clinton, E., Mcadam, M., \& Gamble, J. R. (2018). Transgenerational entrepreneurial family firms: An examination of the business model construct. Journal of Business Research, 90, 269-285.

Cruz, A. P. C. D., Frezatti, F., \& Bido, D. D. S. (2015). Estilo de liderança, controle gerencial e inovação: Papel das alavancas de controle. Revista de Administração Contemporânea, 19(6), 772-794.

Dekker, J. C., Lybaert, N., Steijvers, T., Benoît, D., \& Mercken, R. (2012). Family firm types based on the professionalization construct: exploratory research. Family Business Review, 26(1), 81-99.

Della Giustina, K. A., Gasparetto, V., \& João Lunkes, R. (2020). Efeito dos Estilos de Liderança nos Sistemas de Controle Gerencial e no Desempenho Organizacional. Revista Capital Científico, 18(1), 64-81.

Drake, K. D. (2012). Does firm life cycle explain the relation between book-tax differences and earnings persistence? Arizona State University. 
Frezatti, F., Bido, D. S., Cruz, A. P. C., \& Machado, M. J. (2015). Impacts of Interactive and Diagnostic Control System Use on the Innovation Process. BAR - Brazilian Administration Review, 14(3), 1-24.

Gil, A. C. (2002). Como elaborar projetos de pesquisa. São Paulo: Atlas.

Hiebl, M. R. W. (2013). Bean counter or strategist? Differences in the role of the CFO in family and non-family businesses. Journal of Family Business Strategy, 4(2), 147161.

Javed, M., Rashid, M. A., Hussain, G., \& Ali, H. Y. (2020). The effects of corporate social responsibility on corporate reputation and firm financial performance: Moderating role of responsible leadership. Corporate Social Responsibility and Environmental Management. 27(3), 1395-1409.

Kammerland, N., \& Essen, M. V. (2017). Research: family firms are more innovative than other companies, Harvard Business Review Innovation, 1-6.

Miller, D., \& Friesen, P. H. (1984). A longitudinal study of the corporate life cycle. Management Science Journal, 30(10), 1161-1183.

Mitchell, R. K., Agle, B. R., Chrisman, J. J., \& Spence, L. J. (2011). Toward a theory of stakeholder salience in family firms. Business Ethics Quarterly, 21(2), 235-255.

Moreira, L. V. M., \& Frezatti, F. (2019). O papel do sistema de controle gerencial na transição entre estágios do ciclo de vida organizacional em uma empresa familiar. Revista Universo Contábil, 15(1), 65-84.

Necyk, G. A., \& Frezatti, F. (2010). A Contabilidade Gerencial: uma perspectiva de ciclo de vida de seu desenvolvimento nas organizações. Organizações \& Sociedade, 17(8), 725-744.

Nisiyama, E. K., \& Oyadomari, J. C. T. (2012). Management Control Systems and the Innovation Process. RAI Revista de Administração e Inovação, 9(1), 106-125.

Oro, I. M., \& Lavarda, C. E. F. (2019). Interface between management control systems and strategy and performance measures in a family business. Revista Contabilidade \& Finanças, 30(79), 14-27.

Pimentel, D. (2014). Defining and Characterizing Family Business in an Ultra-Peripheral Economic Context: Terceira Island, Azores. In Proceedings of the 20th APDR Congress. 
Pimentel, D. (2018). Non-family employees: levels of job satisfaction and organizational justice in small and medium-sized family and non-family firms. European Journal of Family Business, 8(2), 93-102.

Raupp, F. M., Martins, S. J., \& Beuren, I. M. (2006). Utilização de controles de gestão nas maiores indústrias catarinenses. Revista Contabilidade \& Finanças, 17(40), 120-132.

Sebrae, Serviço Brasileiro de Apoio às Micro e Pequenas Empresas. Sucessão familiar: mantendo o negócio em família. (2020). Recuperado de: < https://sebrae.ms/empreendedorismo/sucessao-familiar/\#s. Acesso em: 10/agosto/2020.

Sebrae, Serviço Brasileiro de Apoio às Micro e Pequenas Empresas. Porte do Comércio, Indústria e Serviços. Porte das empresas. (2017). Recuperado de: <https://m.sebrae.com.br/Sebrae/Portal\%20Sebrae/UFs/SP/Pesquisas/MPE_concei to_empregados.pdf>. Acesso em: 10 de julho de 2020.

Segura, L. C., \& Formigoni, H. (2014). Influência do Controle e da Gestão Familiar no Endividamento das Empresas Abertas Brasileiras: um Estudo Quantitativo. Brazilian Business Review. 11(6), 51-76.

Sgrò, F., Palazzi, F., Ciambotti, M., \& Gelsomini, L. (2020). Factors promoting and hindering the adoption of management accounting tools. Evidence from Italian manufacturing SMEs. Management Control, 1, 19-38.

Silva, A., \& Beuren, I. M. (2020). Efeitos dos sistemas de controle gerencial e do compartilhamento de informações no risco interorganizacional de uma rede de franquia. Advances in Scientific and Applied Accounting, 13(1), 03-22.

Simons, R. (1994). Levers of control: How managers use innovative control systems to drive strategic renewal. Harvard Business Press.

Songini, L., Gnan, L., \& Malmi, T. (2013). The role and impact of accounting in family business. Journal of Family Business Strategy, 4(2), 71-83.

Su, S., Baird, K., \& Schoch, H. (2015). The moderating effect of organisational life cycle stages on the association between the interactive and diagnostic approaches to using controls with organisational performance. Management Accounting Research, 26, 40-53.

Truant, E., Broccardo, L., \& Vanessa, V. (2019). Sistemas de controle gerencial em empresas familiares: as mulheres são importantes? Evidências do setor alimentar 
italiano. Revista Internacional de Empreendedorismo e Pequenas Empresas, 37(1), 54-70.

Vinagre, L. M., Silva, S. W., Portugal, P. S., Souza, R. S., Gonçalves, J. E., \& Pereira, W. F. (2017). O processo sucessório em uma empresa familiar: um estudo de caso em um supermercado de Varginha-MG. Revista da Universidade Vale do Rio Verde, 15(1), 689-700.

Wang, Y. Z., Lo, F. Y., \& Weng, S. M. (2019). Family businesses successors knowledge and willingness on sustainable innovation: The moderating role of leader's approval. Journal of Innovation \& Knowledge, 4(3), 188-195.

Yin, R. K. (2005). Estudo de Caso: Planejamento e Métodos, (5a ed.). Porto Alegre, Bookma.

Data de Submissão: 19/10/2020

Data de Aceite: 16/12/2020 